\title{
ARTICLE OPEN \\ Plant-derived virus-like particle vaccines drive cross-presentation of influenza A hemagglutinin peptides by human monocyte-derived macrophages
}

Alexander I. Makarkov (iD ${ }^{1,2,11}$, Makan Golizeh (iD ${ }^{2}$, Elizabeth Ruiz-Lancheros ${ }^{2}$, Angelica A. Gopal ${ }^{3,4}$, lan N. Costas-Cancelas ${ }^{5}$, Sabrina Chierzi ${ }^{6}$, Stephane Pillet ${ }^{2,7}$, Nathalie Charland ${ }^{7}$, Nathalie Landry ${ }^{7}$, Isabelle Rouiller $\mathbb{D}^{5,8}$, Paul W. Wiseman (iD ${ }^{3,9}$, Momar $\mathrm{Ndao}^{2,10}$ and Brian J. Ward iD $^{2}$

A growing body of evidence supports the importance of T cell responses to protect against severe influenza, promote viral clearance, and ensure long-term immunity. Plant-derived virus-like particle (VLP) vaccines bearing influenza hemagglutinin (HA) have been shown to elicit strong humoral and $\mathrm{CD}^{+} \mathrm{T}$ cell responses in both pre-clinical and clinical studies. To better understand the immunogenicity of these vaccines, we tracked the intracellular fate of a model HA (A/California/07/2009 H1N1) in human monocyte-derived macrophages (MDMs) following delivery either as VLPs (H1-VLP) or in soluble form. Compared to exposure to soluble HA, pulsing with VLPs resulted in 3-fold greater intracellular accumulation of HA at 15 min that was driven by clathrinmediated and clathrin-independent endocytosis as well as macropinocytosis/phagocytosis. At 45 min, soluble HA had largely disappeared suggesting its handling primarily by high-degradative endosomal pathways. Although the overall fluorescence intensity/cell had declined 25\% at $45 \mathrm{~min}$ after H1-VLP exposure, the endosomal distribution pattern and degree of aggregation suggested that HA delivered by VLP had entered both high-degradative late and low-degradative static early and/or recycling endosomal pathways. At 45 min in the cells pulsed with VLPs, HA was strongly co-localized with Rab5, Rab7, Rab11, MHC II, and MHC I. High-resolution tandem mass spectrometry identified 115 HA-derived peptides associated with MHC I in the H1-VLP-treated MDMs. These data suggest that HA delivery to antigen-presenting cells on plant-derived VLPs facilitates antigen uptake, endosomal processing, and cross-presentation. These observations may help to explain the broad and cross-reactive immune responses generated by these vaccines.

npj Vaccines (2019)4:17; https://doi.org/10.1038/s41541-019-0111-y

\section{INTRODUCTION}

The cellular arm of the adaptive immune response is increasingly recognized as important for both recovery and long-term protection from influenza viruses. CD4 ${ }^{+} \mathrm{T}$ cells provide support for antibody production and maturation as well as the induction of cytotoxic $\mathrm{CD}^{+} \mathrm{T}$ cells (CTL) that target infected cells for elimination. ${ }^{1}$ Although limited in scope, a recent human challenge study with both $\mathrm{H} 1 \mathrm{~N} 1$ and $\mathrm{H} 3 \mathrm{~N} 2$ viruses proposed pre-existing poly-functional $\mathrm{CD}^{+}{ }^{+} \mathrm{T}$ cells as a novel correlate of protection. ${ }^{2}$ Although influenza-specific CTLs cannot prevent disease, they can reduce both the severity and duration of infection. ${ }^{3} \mathrm{~T}$ cell responses may be particularly important for vulnerable populations such as young children and the elderly., ${ }^{4,5}$ The most commonly used influenza vaccines based on detergent-split virions typically elicit a strong antibody response but are weak inducers of cellular immunity. ${ }^{6}$ Although live attenuated vaccines elicit T cell responses, systemic humoral responses are often weak, and interference from pre-existing immunity makes these vaccines less effective after early childhood. ${ }^{7}$ A vaccine that elicits both strong antibody and cell-mediated responses might bring us closer to the control of influenza in the human population.

Plant-derived virus-like particle (VLP) vaccines are produced by Agrobacterium-mediated transient expression of influenza hemagglutinin (HA) proteins in Nicotiana benthamiana. Influenza HAbearing VLPs self-assemble and do not require accessory proteins for budding from the plant cells. ${ }^{8}$ The plant-derived HA-VLPs are $\sim 100 \mathrm{~nm}$ in size. ${ }^{8}$ Each particle has 30-50 homotrimer HA "spikes" inserted into a lipid bilayer envelope of plant cell origin., ${ }^{9,10}$ The HA monomers on the plant-derived VLPs are $\sim 72 \mathrm{kDa}$ corresponding to the uncleaved HAO form. ${ }^{8}$ Plant-derived $\mathrm{H} 1$ proteins contain six $\mathrm{N}$-glycosylation sites, located either in the HA1 globular head or

\footnotetext{
'Division of Experimental Medicine, Department of Medicine, McGill University, 1001 Décarie Street, Montréal, QC H4A 3J1, Canada; ${ }^{2}$ Infectious Diseases and Immunity in Global Health Program, Research Institute of McGill University Health Centre, Glen Site, 1001 Décarie Street, Montréal, QC H4A 3J1, Canada; ${ }^{3}$ Department of Chemistry, McGill University, 801 Sherbrooke St. W, Montreal, QC H3A 0B8, Canada; ${ }^{4}$ Department of Physiology, McGill University, 3655 Promenade Sir William Osler, Montreal, QC H3G 1Y6, Canada; ${ }^{5}$ Department of Anatomy \& Cell Biology, Faculty of Medicine, Groupe de Recherche Axé sur la Structure des Protéines (GRASP), Groupe d'Étude des Protéines Membranaires (GEPROM), McGill University, 3640 University Street, Montreal, QC H3A 2B2, Canada; ${ }^{6}$ Research Institute of McGill University Health Centre, Montreal General Hospital, 1650 Cedar Av, Montréal, QC H3G 1A4, Canada; ${ }^{7}$ Medicago Inc., 1020 Route de l'Église, Bureau 600, Québec, QC G1V 3V9, Canada; ${ }^{8}$ Department of Biochemistry and Molecular Biology and Bio21 Molecular Science and Biotechnology Institute, The University of Melbourne, Parkville, VIC 3010, Australia; ${ }^{9}$ Department of Physics, McGill University, 3600 University St.,

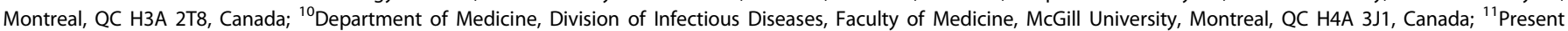
address: Medicago Inc., 1020 Route de I'Église, Bureau 600, Québec, QC G1V 3V9, Canada

Correspondence: Brian J. Ward (brian.ward@mcgill.ca)
}

Received: 20 July 2018 Accepted: 23 April 2019

Published online: 15 May 2019 
the HA2 stem region that carry complex or hybrid glycans containing core $a(1,3)$-fucose or $\beta(1,2)$-xylose epitopes. ${ }^{11}$ Traces of plant proteins can be detected by mass spectrometry (MS), many of which were previously identified in Nicotiana tabacum lipid rafts, suggesting that the mechanism of VLP formation in plants is similar to the natural process of influenza virus assembly in the mammalian host cells.,11 The plant-based transient expression system allows rapid and large-scale production of influenza HAbased vaccine at relatively low cost, addressing several of the challenges for vaccine production in a pandemic (i.e., speed and scalability) and representing an alternative to the currently available manufacturing platforms for seasonal vaccines. ${ }^{8}$ Plantderived VLPs recapitulate the key features of native influenza virions such as sialic acid-mediated adherence and internalization by target cells, fusion of the VLP envelope with endosomal membranes, and rapid induction of an innate immune response. ${ }^{10,12,13}$ These vaccines have been shown to elicit strong and cross-reactive antibody responses against both seasonal and pandemic influenza strains in animal models and human trials. ${ }^{14-}$

${ }^{16}$ They also induce polyfunctional and cross-reactive HA-specific $\mathrm{CD}^{+} \mathrm{T}$ cell responses. ${ }^{14,15,17}$ Simultaneous administration of a plant-derived $\mathrm{H} 1$-VLP vaccine with ovalbumin (OVA) was recently shown to elicit an OVA-specific $\mathrm{CD}^{+}{ }^{+} \mathrm{T}$ cell response in $\mathrm{C} 57 \mathrm{BL} / 6$ mice. ${ }^{18}$ The subcellular mechanisms that account for the unusual immunogenicity of the plant-derived VLP-based vaccines are not yet well understood.

In the current work, we demonstrated that human monocytederived macrophages (MDMs) internalize H1-VLPs using both clathrin-mediated and clathrin-independent endocytosis (CME and $\mathrm{CIE}$ respectively) as well as macropinocytosis and, probably, phagocytosis. Soluble $\mathrm{H} 1$ was internalized almost exclusively by CME and was trafficked predominantly to the high-degradative late endosome/endolysosome compartment. In contrast, a substantial portion of $\mathrm{H} 1$ delivered by VLP was retained in lowdegradative static early and/or recycling endosomes where the HA co-localized with major histocompatibility complex (MHC) I protein. Immunoprecipitation of MHC I and high-resolution MS revealed a large number of $\mathrm{HA}$-derived peptides in MDMs exposed to H1-VLP but not soluble H1. These findings demonstrate that intracellular processing of influenza HA by human MDMs is very different when the protein is delivered by VLP or in a soluble form. These observations help to explain the dual humoral and $\mathrm{CD4}^{+}$responses seen in humans with the plant-derived VLP vaccines and raise the possibility that cross-presentation of $\mathrm{HA}$ peptides to $\mathrm{CD}^{+} \mathrm{T}$ cells may also occur.

\section{RESULTS}

Characterization of influenza HA presented on H1-VLPs and recombinant soluble $\mathrm{H} 1$ protein

To study the uptake and endosomal handling of influenza HA presented on plant-derived H1-VLPs, initial experiments were performed with a commercially available recombinant protein produced in mammalian 293 cells. This comparator consisted of the extracellular and intracellular domains of the $\mathrm{H} 1$ of the $\mathrm{A} /$ California/07/2009 H1N1 virus but lacked the transmembrane portion, which permitted secretion of the soluble protein from host cells and prevented formation of the HA multimeric structures. ${ }^{19}$ SDS-PAGE followed by Coomassie blue staining (Fig. 1a and Supplementary Fig. 1a) and immunoblot analysis with anti$\mathrm{H} 1$ polyclonal (Fig. $1 \mathrm{~b}$ and Supplementary Fig. 1b) or monoclonal (Fig. 1c and Supplementary Fig. 1c) antibodies showed that influenza $\mathrm{H} 1$ was the predominant protein in both H1-VLP and soluble $\mathrm{H} 1$ stocks. HA monomers $\sim 75 \mathrm{kDa}$ (mostly) and HA dimers and trimers, as well as HA1 ( 55 kDa) and HA2 ( 30 kDa) subunits, were observed in $\mathrm{H} 1-\mathrm{VLP}$ samples. The soluble $\mathrm{H} 1$ was present as a single protein $\sim 75 \mathrm{kDa}$ corresponding to uncleaved $\mathrm{HA}$ monomers, as expected. No HA degradation products and no non-specific (non-HA) proteins were found in either the H1-VLP or soluble $\mathrm{H} 1$ samples. The monoclonal anti-H1 antibody that we used in imaging experiments (both confocal and transmission electron microscopy-TEM) bound equally well to HA in both $\mathrm{H1}$ VLPs and the soluble $\mathrm{H} 1$ comparator (Fig. 1c and Supplementary Fig. 1c). The antibody binding epitope was located on the HA2 subunit, as revealed by visualization of uncleaved HA and $\sim 30 \mathrm{kDa}$ (but not $\sim 55 \mathrm{kDa}$ ) bands on the H1-VLP immunoblot. The protein concentration of both products measured by BCA assay was consistent with the HA content reported by the respective manufacturers. Negative stain TEM of H1-VLPs showed pleomorphic particles with an average diameter of $\sim 100 \mathrm{~nm}$ and spikes on their surface (Supplementary Fig. 1d). VLP-like objects were observed on the surface of MDMs at 5 min after exposure to H1-VLPs but not the soluble H1 (Supplementary Fig. 1e). Nanogold immunostaining with anti-H1 antibody confirmed that these objects were indeed the HA-bearing VLPs. Nanogold-labeled HA was found on the surface of VLP-treated MDMs (Fig. 1d) and inside the endosomes (Fig. 1e).

In summary, plant-derived VLPs bearing influenza HA are pleomorphic particulate structures, similar in size to native influenza virions. The HA molecules on the H1-VLPs form supramolecular complexes, whereas the comparator (soluble $\mathrm{H} 1$ protein) is monomeric. Both the $\mathrm{H} 1-\mathrm{VLP}$ and soluble $\mathrm{H} 1$ preparations contained primarily HA protein without any unexpected degradation products or non-HA contaminants. The anti$\mathrm{H} 1$ monoclonal antibody used in these studies bound equally well to the HA from both sources.

\section{H1-VLPs are efficiently internalized by human MDMs}

Classical TEM was used to document early endocytotic events such as formation of endocytic vesicles that are too small to be well-visualized with confocal microscopy. ${ }^{20}$ Exposure of MDMs to $\mathrm{H} 1$-VLPs led to rapid activation of the endocytosis machinery. The number of endocytic vesicles doubled during the first 5 min of exposure to H1-VLPs while soluble $\mathrm{H} 1$ had no significant effect (Fig. 2a). Intracellular HA immunofluorescence was apparent at 5 min of exposure to H1-VLPs and reached a plateau at $10 \mathrm{~min}$. Further incubation did not change the fluorescence signal (Supplementary Fig. 2a). To eliminate continuous internalization, MDMs were pulsed with either H1-VLPs or soluble $\mathrm{H} 1 \mathrm{for} 15 \mathrm{~min}$ followed by a $30 \mathrm{~min}$ incubation. At $15 \mathrm{~min}$, the fluorescence was 3-fold higher in H1-VLP-treated MDMs compared to soluble H1 (Fig. 2b). Of note, the HA fluorescence intensity in MDMs exposed to H1-VLPs was $\sim 4-6$-fold greater than in those exposed to the licensed monovalent split virion $\mathrm{H} 1 \mathrm{~N} 1$ vaccine (Supplementary Fig. 2b), suggesting that recombinant soluble HA and HA from the split virion vaccine display similar MDM internalization characteristics, and that soluble HA can serve as a comparator to study the particulate form of HA (i.e., VLPs). Next, we used a 1,1'-dioctadecyl3,3,3',3'-tetramethylindodicarbocyanine perchlorate (DiD) dequenching assay ${ }^{13}$ and a panel of endocytosis inhibitors (Supplementary Table 1 and Supplementary Fig. 2c-e) to demonstrate that $\mathrm{H} 1$-VLPs were internalized primarily through $\mathrm{CME}$ and $\mathrm{CIE}$ with smaller contributions from macropinocytosis and, probably, phagocytosis (Fig. 2c). The prominent role of CME was further demonstrated by direct $\mathrm{HA}$ immunofluorescence in the presence of CME inhibitor chlorpromazine (Fig. 2d) and by colocalization of HA with fluorescently-labeled transferrin that is exclusively taken up by CME (Fig. 2e). Immunolabeling of clathrin and caveolin-1 in TEM images of MDMs exposed to H1-VLPs confirmed that the total endocytic vesicle pool included both clathrin-coated and caveolin-coated structures (Fig. 2f). Soluble H1 endocytosis was largely unaffected by the CIE inhibitor genistein but was greatly reduced by chlorpromazine (Fig. $2 \mathrm{~d}$ ). Internalized 
a

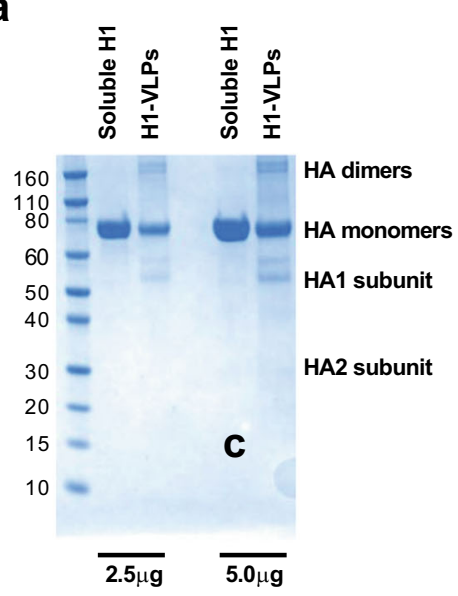

d

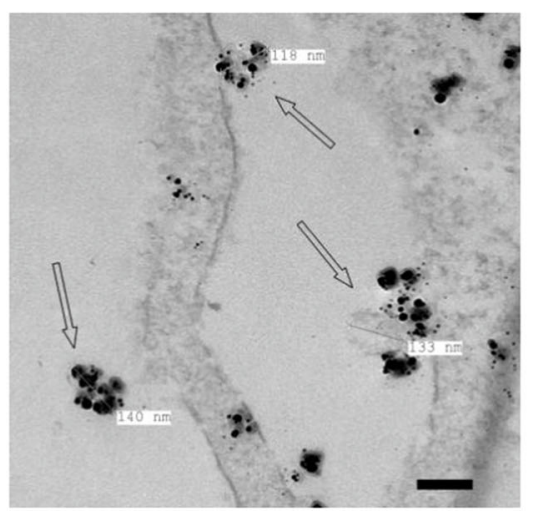

b

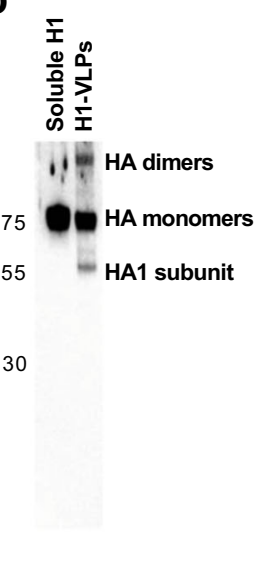

C

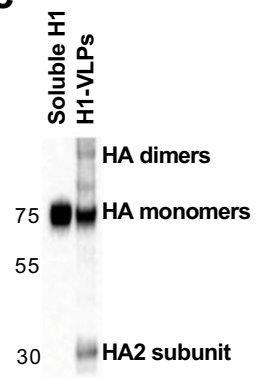

e

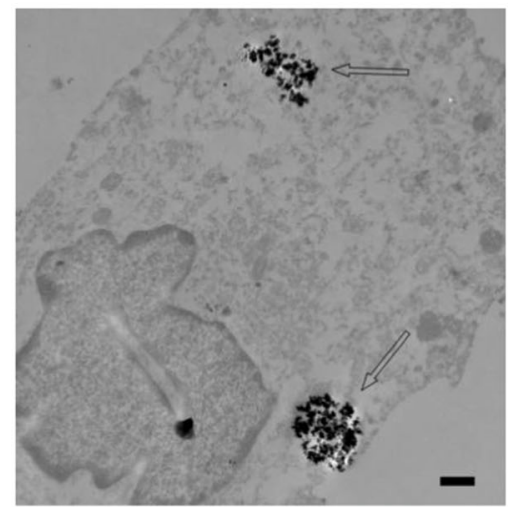

Fig. 1 Characterization of influenza HA presented on H1-VLPs and recombinant soluble H1 protein. a SDS-PAGE of H1-VLPS and soluble H1 protein $(2.5$ and $5.0 \mu \mathrm{g}$ per sample) followed by Coomassie blue staining. Representative image from 3 experiments shown. b Immunoblot analysis of $\mathrm{H} 1-\mathrm{VLPs}$ and soluble $\mathrm{H} 1$ protein using rabbit polyclonal anti-H1 antibody. Representative image from 3 experiments shown. c Immunoblot analysis of $\mathrm{H} 1-\mathrm{VLPs}$ and soluble $\mathrm{H} 1$ protein using mouse monoclonal anti-H1 antibody. Representative image from 3 experiments shown. d Representative TEM image shows H1-VLPs with nanogold immunolabeled HA. Arrows indicate HA-bearing VLPs surrounded by nanogold particles on the surface of MDM exposed to H1-VLPs. Scale bar-100 nm. e Representative TEM image shows endosomal accumulation of nanogold immunolabeled HA in MDM. Arrows indicate HA-loaded endosomes in MDM exposed to H1-VLPs for $15 \mathrm{~min}$. Scale bar-500 $\mathrm{nm}$

soluble HA was almost perfectly co-localized with transferrin (Fig. 2e).

In summary, MDMs exposed to H1-VLPs internalized much more HA compared to those pulsed with soluble H1. H1-VLP internalization occurred through multiple endocytic pathways including CME, $\mathrm{CIE}$, macropinocytosis and, probably, phagocytosis while soluble $\mathrm{H} 1$ was internalized almost exclusively by CME. The diversity of $\mathrm{H} 1-$ VLPs internalization mechanisms raised the possibility that antigen delivered in this form might experience different acidification and degradative environments, leading to a broader range of antigen processing and presentation pathways. ${ }^{21}$ It was therefore of interest to study endosomal trafficking and the intracellular fate of the two forms of HA.

H1-VLPs are handled in two distinct endosomal pools: high- and low-degradative

Both the amount of internalized $\mathrm{HA}$ and the degree of degradation over time varied with delivery form (Fig. 3a and Supplementary Fig. 3a). We used the intensity of HA fluorescence as a surrogate for protein degradation, assuming that the monoclonal antibody-binding epitope would be preserved in a low-degradative intracellular compartment (early-static or recycling endosomes). Conversely, the disappearance of the fluorescent signal would suggest trafficking to late endosomes/ endolysosomes. Using confocal microscopy, the intensity of HA fluorescence in MDMs pulsed with soluble $\mathrm{H} 1$ dropped dramatically over $45 \mathrm{~min}$ (>90\%) while the cells pulsed with H1-VLP retained $\sim 75 \%$ of the $\mathrm{HA}$ signal, suggesting that a substantial portion of the internalized protein delivered by VLP had found its way into low-degradative cellular compartments. To more precisely define the fate of the internalized HA, we analyzed the confocal data using the fluorescence fluctuation method image cross-correlation spectroscopy (ICCS) with segmentation. ${ }^{22,23} \mathrm{We}$ based the segmentation on HA-positive endosomes (Supplementary Fig. 3b) via automatic thresholding based on the fluorescence intensity. The intensity of HA fluorescence in the endosomes of VLP-pulsed cells increased more than two-fold between 15 and $45 \mathrm{~min}$. The cluster density (number of HA particles per $\mu \mathrm{m}^{2}$ ) and the degree of HA aggregation also increased (Fig. 3b). In contrast, HA fluorescence intensity fell $\sim 97 \%$ in the endosomes of MDMs pulsed with soluble $\mathrm{H} 1$ during this same time period. The cluster density and the degree of HA aggregation in these samples was greatly reduced as well, suggesting that the internalized soluble protein was almost completely degraded by $45 \mathrm{~min}$.

Thus, exposure of MDMs to H1-VLPs resulted in rapid and substantial endocytosis. A large proportion of HA remained intact for at least $45 \mathrm{~min}$ after the VLP pulse. Moreover, increases in HA fluorescence intensity, cluster density, and degree of aggregation all 
a

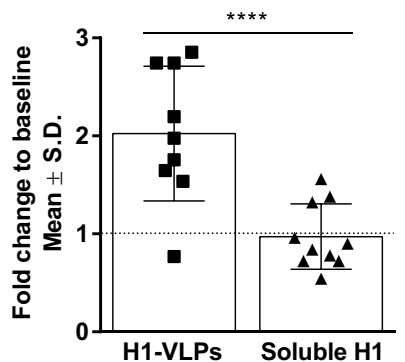

b

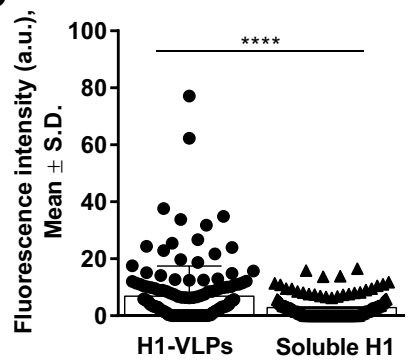

C

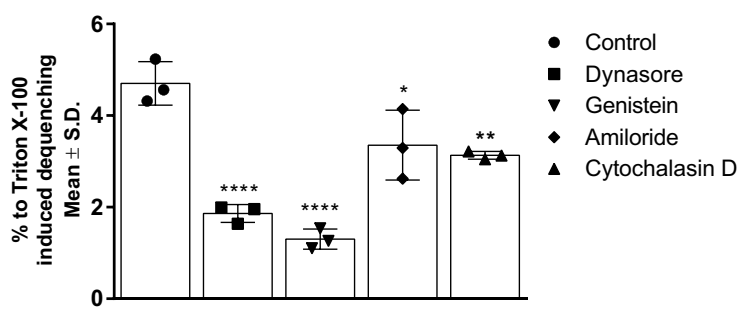

d

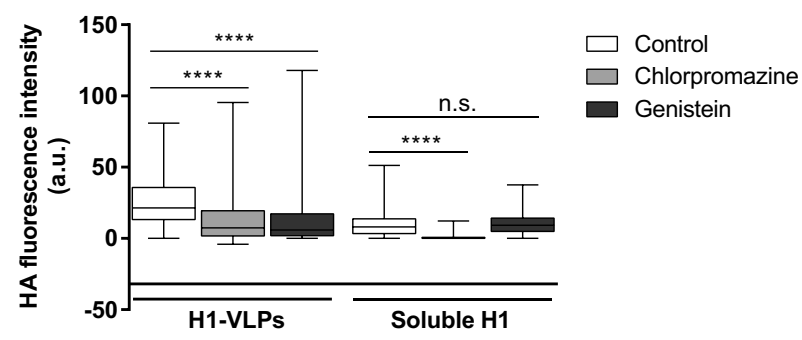

e

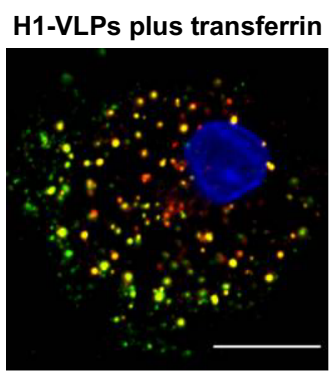

Soluble $\mathrm{H} 1$ plus transferrin
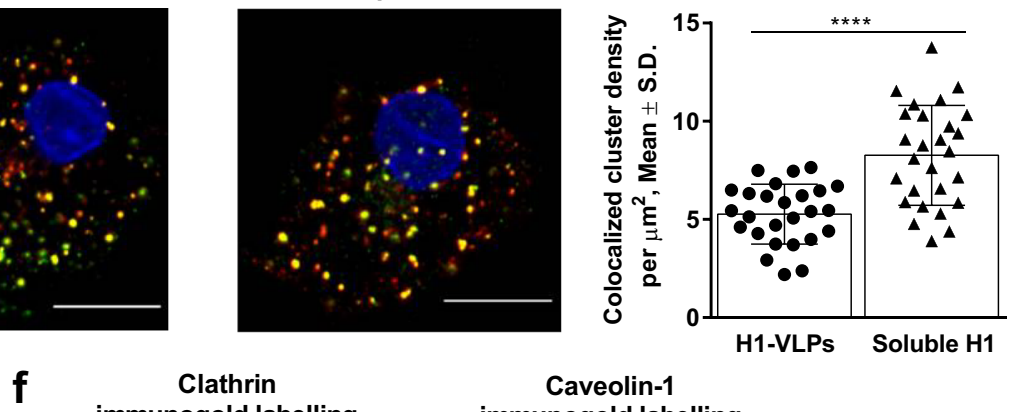

Clathrin

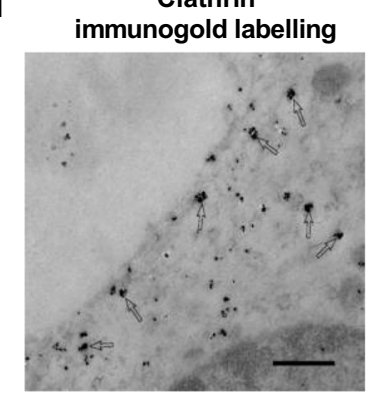

Caveolin-1 immunogold labelling

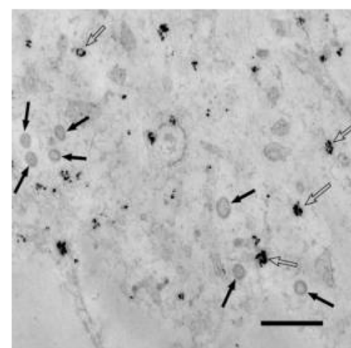

Fig. 2 Mechanisms of $\mathrm{H} 1$-VLPs and soluble $\mathrm{H} 1$ internalization by human MDMs. a Number of endocytic vesicles in MDMs exposed to H1-VLPs or soluble $\mathrm{H} 1$ for $5 \mathrm{~min}$, normalized against the baseline count (taken as 1, dotted line). Data from two experiments were analyzed. b HA internalization by MDMs exposed to either H1-VLPs or soluble $\mathrm{H} 1$ for $15 \mathrm{~min}$. The amount of internalized protein was evaluated by the intensity of HA immunofluorescence per cell area on confocal microscopy images. Based on 4 experiments. c Effect of endocytosis inhibitors on DiD dequenching by MDMs loaded with DiD-labeled H1-VLPs (at $2 \mathrm{~h}$ ). Data from 3 experiments were analyzed. $\mathbf{d}$ Effect of chlorpromazine and genistein on $\mathrm{H} 1$-VLPs or soluble $\mathrm{H} 1$ internalization by MDMs upon $15 \mathrm{~min}$ of exposure. The amount of internalized protein evaluated by the intensity of HA immunofluorescence per cell area on confocal microscopy images. Based on 6 experiments. e Colocalization of HA and transferrin in MDMs exposed to H1-VLPs and transferrin (left) or soluble H1 and transferrin (center), and segmentation ICCS colocalization (number of colocalized particles per $\mu \mathrm{m}^{2}$-right). Representative images from 3 experiments shown. Scale bar-10 $\mu \mathrm{m}$. Green: fluorescently labeled HA, red: transferrin conjugated with CF568 fluorophore (yellow shows colocalization of two proteins), blue: nuclei stained with DAPI. f Representative TEM image with nanogold immunolabeled clathrin (left). Open arrows indicate clathrin-coated endocytic vesicles. Representative TEM image with nanogold immunolabeled caveolin-1 (right). Open arrows indicate caveolin-coated endocytic vesicles. Solid arrows indicate unlabeled clathrin-coated endocytic vesicles with typical clathrin spikes. Scale bar-500 nm. Bar graphs present mean \pm standard deviation (S.D.); box and whisker plot presents the minimum, maximum, median, and 25 th and 75 th percentiles. ${ }^{*} p<0.05$, ${ }^{* *} p<0.01$, ${ }^{* * * *} p<0.0001$. n.s.: nonsignificant (a, $\mathbf{c}, \mathbf{d}$-one-way ANOVA followed by Tukey's multiple comparisons post-test; $\mathbf{b}$, e-Mann-Whitney test)

suggested homotypic fusion of the HA-positive endosomes. ${ }^{24}$ The simultaneous reduction in overall HA fluorescence intensity per cell area argued for movement of some of the protein to high- degradative late endosomes, supporting a bidirectional trafficking model for the HA delivered by the VLPs. In contrast, the uptake of soluble $\mathrm{H} 1$ was less substantial at the outset $(15 \mathrm{~min})$ and the HA 
a

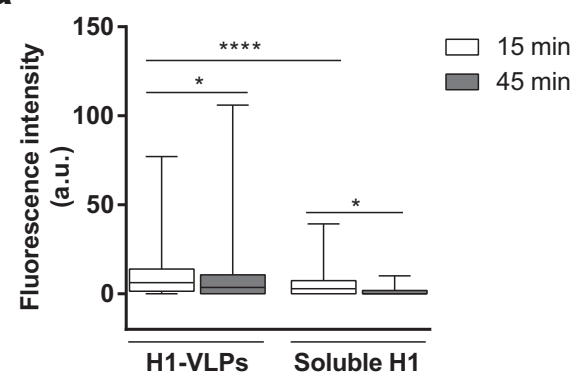

b
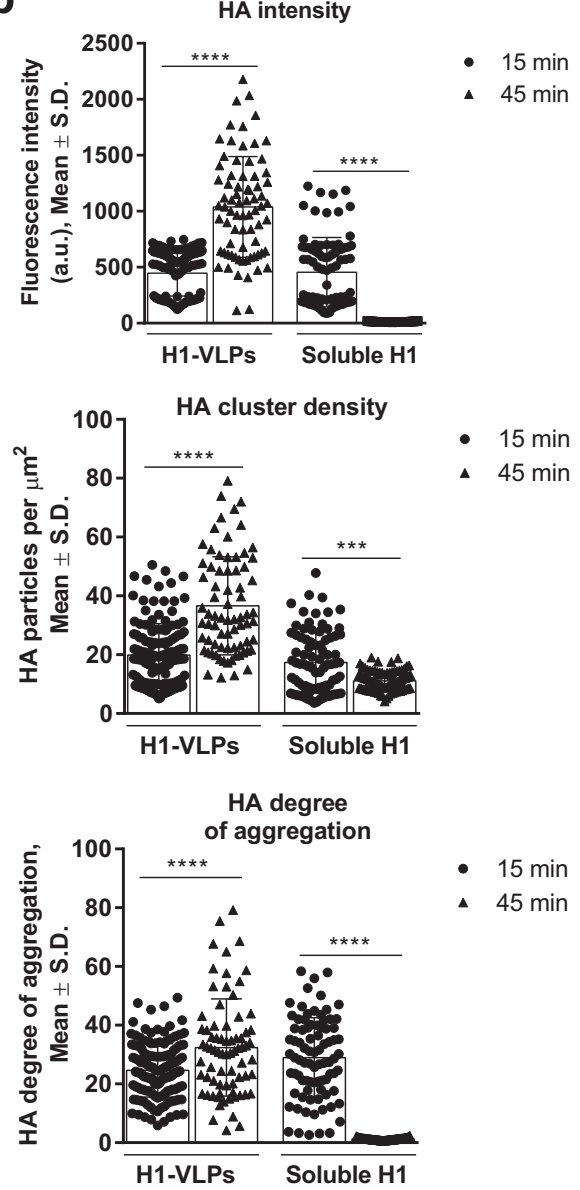

Fig. 3 Intracellular HA distribution in human MDMs exposed to $\mathrm{H} 1-$ VLPs or soluble H1. a HA internalization and degradation by MDMs pulsed with either H1-VLPs or soluble $\mathrm{H} 1$. The amount of internalized protein evaluated by the intensity of HA immunofluorescence per cell area on confocal microscopy images. Data from 9 experiments were analyzed. b Segmentation ICCS analysis of the HA endosomal distribution in MDMs pulsed $(15 \mathrm{~min}$ ) with either $\mathrm{H} 1$ VLPs or soluble H1 shows HA fluorescence intensity (top), cluster density (number of fluorescent particles per $\mu \mathrm{m}^{2}$-middle) and degree of HA aggregation (bottom). Based on 7 experiments. Bar graphs present mean \pm standard deviation (S.D.); box and whisker plot presents the minimum, maximum, median, and 25th and 75th percentiles. ${ }^{*} p<0.05,{ }^{* *} p<0.001,{ }^{* * * *} p<0.0001$ (one-way ANOVA followed by Tukey's multiple comparisons post-test)

fluorescence had almost completely disappeared at $45 \mathrm{~min}$. These observations prompted us to further characterize the endosomal compartments contributing to the complex handling of $\mathrm{HA}$ delivered on VLPs or as soluble protein.
H1-VLPs move towards static early and/or recycling endosomes in human MDMs

Conventional colocalization analysis based on "per cell" image segmentation suggested that H1-VLPs preferentially track from early, Rab5-positive endosomes to low-degradative Rab11-positive recycling endosomes rather than to late Rab7-positive endosomes/endolysosomes (Supplementary Fig. 4a). ${ }^{25}$ Of note, in a significant minority of H1-VLP-exposed cells ( 15\%), we observed peripheral re-distribution (recycling) of undegraded HA towards the plasma membrane at $45 \mathrm{~min}$ (Supplementary Fig. 4b). Soluble $\mathrm{H} 1$ was partially co-localized with all three endosomal markers at 15 min but was undetectable in any endosomal compartment by 45 min, suggesting that the HA had been almost fully degraded. When the HA-positive endosomal compartment was characterized by segmentation ICCS analysis, there was a substantial increase in the HA colocalized cluster density (number of colocalized particles per $\mu \mathrm{m}^{2}$ ) with Rab5 and Rab11 markers at $45 \mathrm{~min}$, suggesting protein retention in static early and/or recycling endosomes (Fig. 4a). Unexpectedly, we also observed an increase of HA-Rab7 colocalized cluster density, which may possibly be explained by Rab conversion of the slowly-maturing endosomes. ${ }^{26}$ The HA-MHC II colocalized cluster density was $\sim 4$-fold higher in the $\mathrm{H} 1$-VLP-pulsed MDMs compared to those treated with soluble $\mathrm{H} 1$ at $15 \mathrm{~min}$, and did not change by $45 \mathrm{~min}$ (Fig. 4b), suggesting that VLPs facilitate HA delivery, at least partially, in high-degradative Rab7 $^{+}$compartments that favor MHC II-restricted antigen presentation. $^{21,27}$ The fraction of HA particles interacting with endosomal proteins remained unchanged between 15 and $45 \mathrm{~min}$ in the MDMs exposed to H1-VLPs (Fig. 4), suggesting that HAenriched endosomes retained association with Rab proteins that regulate endosomal trafficking, cargo sorting, and organelle maturation. ${ }^{28}$

In summary, a large portion of the HA delivered on VLPs is retained in low-degradative endosomal compartments (static early and/or recycling endosomes) for at least $45 \mathrm{~min}$, while the remainder follows the "classic" endosomal degradation pathway. In contrast, soluble $\mathrm{H} 1$ is mostly trafficked towards highdegradative intracellular compartments. These striking differences in the intracellular handling of HA raised questions about the possible immunological consequences of the two forms of antigen delivery.

HA delivery in the form of VLPs favors antigen cross-presentation by human MDMs

A large portion of the intracellular $\mathrm{MHC}$ I pool resides in recycling Rab11a-positive endosomes that can support cross-presentation of phagocytosed antigens. ${ }^{29}$ It was therefore of interest to explore $\mathrm{HA}$ delivery into the MHC I-positive endosomal compartments following pulsing of MDMs with the different forms of HA. ICCS colocalization revealed a strong association between $\mathrm{HA}$ and $\mathrm{MHC}$ I molecules in MDMs pulsed with either H1-VLPs or soluble $\mathrm{H} 1$ at 15 min (Fig. 5a). By 45 min however, HA-MHC I colocalized cluster density had greatly increased in H1-VLP-exposed cells (213\%) but fell by $63 \%$ in the MDMs pulsed with soluble HA. The fraction of interacting $\mathrm{HA}$ particles remained unchanged by $45 \mathrm{~min}$ in $\mathrm{H1}$ VLP-exposed MDMs. Based on the assumption that prolonged retention of antigen in low-degradative $\left(\mathrm{MHC} \mathrm{I} \mathrm{I}^{+}, \mathrm{Rab}_{11^{+}}\right.$) compartments favors cross-presentation, ${ }^{30}$ we immunoprecipitated MHC I-peptide complexes from lysates of MDMs that had been pulsed overnight with H1-VLPs or soluble $\mathrm{H} 1$ and analyzed the eluted peptides using high-resolution tandem MS. No confident HA-derived peptides were detected in the lysate of MDMs exposed to soluble $\mathrm{H} 1$. In contrast, $115 \mathrm{HA}$-derived $\mathrm{MHC} \mathrm{I-}$ associated peptides were identified in MDMs exposed to the $\mathrm{H} 1$ VLPs (posterior error probability (PEP) score $\leq 0.01$ ), contributing to an HA sequence coverage of $12-89 \%$ (Table 1 and Supplementary 
a
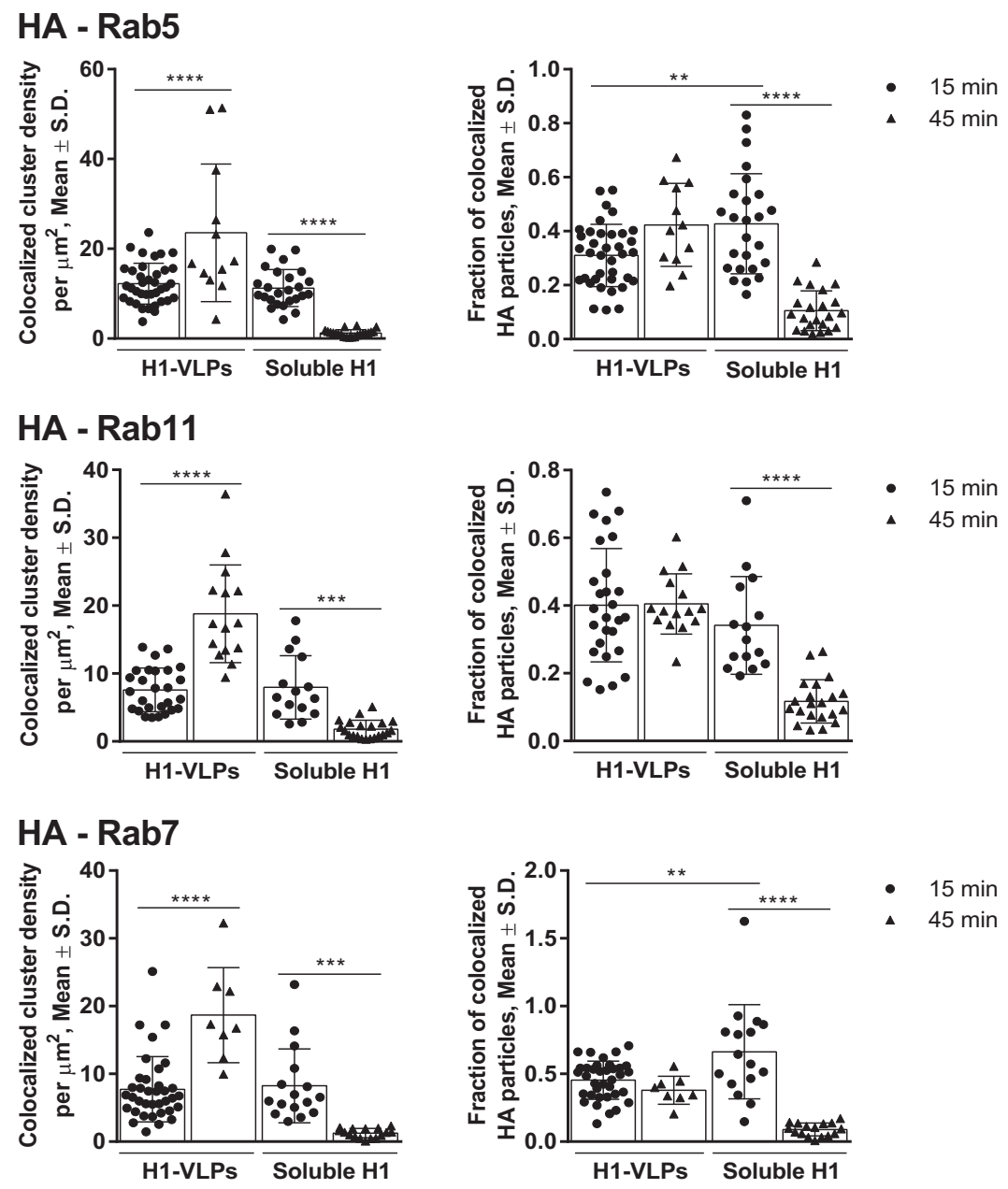

b
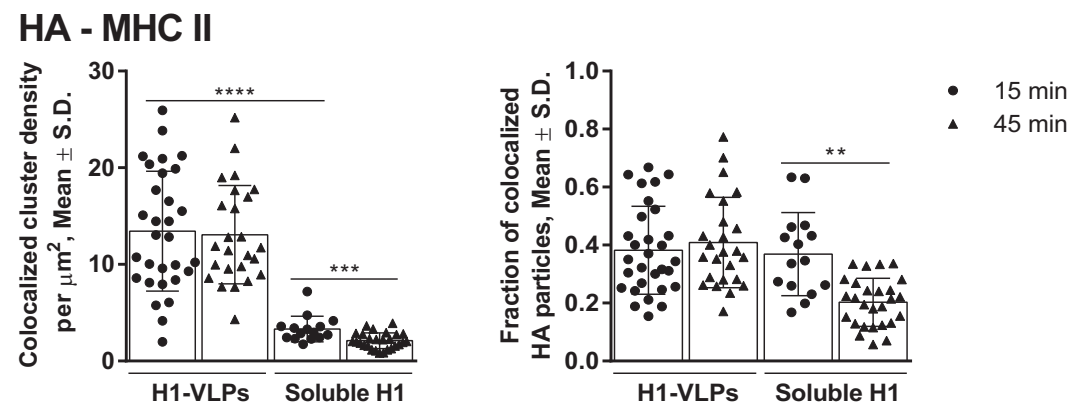

Fig. 4 Segmentation ICCS analysis of HA colocalization with Rab proteins and MHC II. a HA colocalization (number of colocalized particles per $\mu \mathrm{m}^{2}$ - left) and colocalized fraction of HA (right) with Rab5 (top), Rab11 (middle), and Rab7 (bottom) are presented. Based on 3 or more experiments for each condition. b HA colocalization (number of colocalized particles per $\mu \mathrm{m}^{2}$-left) and colocalized fraction of HA (right) with MHC II are presented. Based on three experiments. Bar graphs present mean \pm standard deviation (S.D.). ${ }^{* *} p<0.01,{ }^{* * *} p<0.001,{ }^{* * * *} p<0.0001$ (one-way ANOVA followed by Tukey's multiple comparisons post-test)

Table 2). Eight peptides were detected in more than one donor and the HA protein scores varied from 32 to 323 . Among the 115 HA-derived peptides, 50 (43.5\%) came from the globular head, 51 $(44.3 \%)$ from the stem domain, and $14(12.2 \%)$ from the transmembrane/intracellular portion of HA. Average protein quantity in cell lysates did not differ between HA treatment groups $(2773.8 \pm 595.2 \mu \mathrm{g} / \mathrm{mL}$ in VLP group and $2440.4 \pm 468.1 \mu \mathrm{g} /$ $\mathrm{mL}$ in soluble HA group; $p=0.74$; Mann-Whitney test). The HAderived peptides averaged 18 amino acids (AA) and only $10 \%$ had an "optimal" length for MHC I loading (8-10 AA: Fig. 5b). ${ }^{30}$
This observation suggested that the lysates contained a mixture of optimally-trimmed peptides and immature peptides from the endoplasmic reticulum (ER)/endosome compartments still being processed and sorted for either presentation or degradation. ${ }^{31}$

\section{DISCUSSION}

As bridges between innate and adaptive immune responses, "professional" phagocytes such as macrophages are first-line defenders against invading pathogens. ${ }^{32}$ Among other activities, 
a
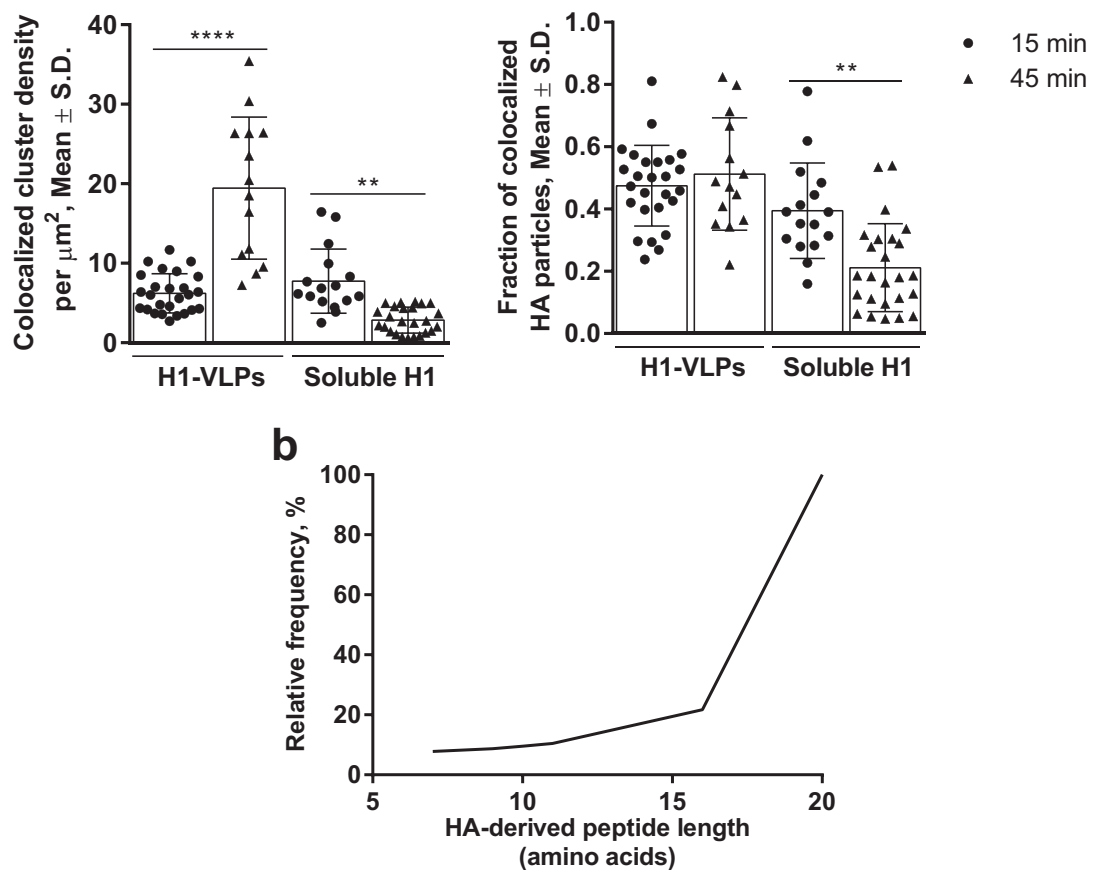

Fig. 5 HA cross-presentation by human MDMs exposed to H1-VLPs. a Segmentation ICCS analysis of HA colocalization with MHC I. The colocalization (number of colocalized particles per $\mu \mathrm{m}^{2}$-left) and colocalized fraction of HA (right) with MHC I presented. Based on 3 experiments. Bar graphs present mean \pm standard deviation (S.D.). ${ }^{* *} p<0.01,{ }^{* * *} p<0.0001$ (one-way ANOVA followed by Tukey's multiple comparisons post-test). $\mathbf{b}$ Cumulative curve shows the distribution by length (number of amino acids) of the HA-derived peptides detected from H1-VLP-treated MDMs

Table 1. Mass spectrometry analysis of HA-derived peptides from $\mathrm{H} 1$ VLP or soluble H1-treated MDMs obtained from four donors

\begin{tabular}{lllll}
\hline Parameters & Donor A & Donor B & Donor C & Donor D \\
\hline HA treatment & Soluble H1 & - & Soluble H1 & Soluble H1 \\
Total protein amount, $\mu \mathrm{g}$ & 2397 & - & 1652 & 3272 \\
Sequence coverage (\%) & 0 & - & 0 & 0 \\
Protein score & 0 & - & 0 & 0 \\
\# MS/MS spectra & 0 & - & 0 & 0 \\
\# Peptides & 0 & - & 0 & 0 \\
HA treatment & H1-VLPs & H1-VLPs & H1-VLPs & H1-VLPs \\
Total protein amount, $\mu$ g & 2653 & 1444 & 2658 & 4340 \\
Sequence coverage (\%) & 61 & 12 & 30 & 89 \\
Protein score & 32 & 75 & 152 & 323 \\
\# MS/MS spectra & 29 & 11 & 16 & 84 \\
\# Peptides & 25 & 10 & 14 & 66 \\
\hline
\end{tabular}

antigen uptake, processing, and display by these cells contribute to both the strength and the pattern of the immune response. In this in vitro work, we focused on human MDMs that phenotypically and functionally resemble the inflammatory-type macrophages $^{33}$ implicated in orchestrating early responses to influenza virus infection. ${ }^{34}$ Our goal was to better understand how these cells handle influenza HA proteins when delivered either as a soluble protein (i.e., in the form of single molecules or small supramolecular complexes) or decorating the surface of $\sim 100 \mathrm{~nm}$ plant-derived VLPs. HA was presented on the surface of VLPs in the form of trimers whereas soluble HA that lacks the transmembrane portion was delivered in the monomeric form. ${ }^{19}$
Since no difference was observed in the uptake of monovalent $\mathrm{H} 1 \mathrm{~N} 1$ influenza vaccine and recombinant soluble $\mathrm{H} 1$ (Fig. $2 \mathrm{~b}$ and Supplementary Fig. 2b), we used the latter in these experiments in order to minimize possible biases introduced by the presence of influenza proteins other than $H A$, viral RNA, egg-derived components, detergent, and formaldehyde traces in the split virion vaccine.

Regardless of the production platform, VLPs are nanoparticles bearing viral antigens that resemble native virions in size and, in many cases, structure. ${ }^{10}$ VLPs are often highly immunogenic, but are non-infectious due to lack of the genetic material. ${ }^{35-37}$ Several very successful VLP vaccines based on recombinant antigens are in current use including those targeting hepatitis B virus and human papillomaviruses. ${ }^{38}$ VLPs can display a number of antigens with or without adjuvant molecules, and therefore have the potential to elicit broad immune responses. ${ }^{36}$ VLPs typically retain the native conformation of the antigens they display; they are organized in an ordered array and in a particulate form, all of which promote strong immune responses. ${ }^{36,39}$ Due to highly repetitive epitopes on their surface, VLPs can act as strong activators of antigen-presenting cells (APCs), facilitating internalization and presentation of antigens, resulting in strong induction of antibodies as well as virus-specific $\mathrm{CD}^{+}$and $\mathrm{CD} 8^{+}$ T cells. ${ }^{40,41}$ A number of VLP-based vaccine candidates expressing influenza HA with or without other viral proteins are under development. ${ }^{42-49}$

VLPs can be produced in bacteria (Escherichia coli) ${ }^{50}$ or yeast, ${ }^{51}$ in insect, ${ }^{52}$ mammalian $^{53}$ and plant cells $s^{8}$ and even in cell-free systems in vitro. ${ }^{54}$ Production in plants is attractive for several reasons, ${ }^{55}$ including low cost and scalability. Furthermore, expression of candidate vaccines in plants exploits their eukaryotic processing machinery, supporting appropriate post-translational modifications and assembly of antigens. Finally, plant-derived 
VLPs may have a significant safety advantage since the risk of contamination with human pathogens is extremely low. ${ }^{56-58}$ Plant-derived influenza vaccine candidate VLPs have been successfully produced by a number of academic and industrial groups. ${ }^{8,59,60}$

We have previously shown that plant-derived VLPs rapidly interact with human immune cells including B cells, monocytes, and dendritic cells ${ }^{10,12,18}$ and that early interactions with human MDMs (i.e., binding, internalization, entry into endosomes and fusion with endosomal membranes) are similar to what happens with wild-type influenza virions. ${ }^{13}$ The efficiency of the VLP-MDM interactions may largely be attributable to the particulate form of the VLPs bearing 30-50 HA trimers that stimulate rapid binding and internalization. ${ }^{10,13}$ In the current work, we tracked intracellular handling by MDMs at much higher resolution and demonstrated that a large proportion of the HA delivered on these VLPs enters static and recycling endosomal pathways leading to $\mathrm{MHC}$ I cross-presentation.

Many factors contribute to how APCs handle any given antigen including the dose and form of the antigen itself, the nature and activation state of the APCs as well as the microenvironment in which these processes occur. How the antigen first enters the cell can also strongly influence the outcome and APCs have many choices from "bulk" processes like phagocytosis and macropinocytosis or more controlled processes like CME or CIE. Antigens internalized through CME are usually delivered rapidly to maturing degradative endosomes that undergo quick acidification and fusion with lysosomes. ${ }^{61}$ The cleavage products of lysosomal proteases and peptidases are typically longer peptides (13-25 AA) appropriate for MHC II loading that leads to priming of $\mathrm{CD}^{+}$ $\mathrm{T}$ cells and support of strong humoral responses. ${ }^{27}$ In our studies, H1-VLP-pulsed MDMs demonstrated substantially greater $\mathrm{HA}-\mathrm{MHC}$ II colocalization than soluble $\mathrm{H} 1$-treated cells, suggesting that the VLPs may favor, at least in part, MHC II-restricted presentation of HA-derived peptides. We also observed nearly complete disappearance of soluble $\mathrm{H} 1$ immunofluorescence at $45 \mathrm{~min}$, suggesting predominant trafficking towards the highdegradative endolysosomal compartment in human MDMs. Such handling is certainly consistent with the observation that split virion influenza vaccines typically elicit strong antibody responses but little-to-no priming of $\mathrm{CD}^{+}{ }^{+} \mathrm{T}$ cells and only limited cellmediated immunity against influenza. ${ }^{6}$

In contrast, CIE often leads to homotypic fusion of caveolincoated endocytic vesicles and formation of large caveosomes that can retain non-degraded antigenic material for long periods of time. ${ }^{62}$ Although the mechanisms are not yet fully understood, both static early endosomes ${ }^{63}$ and non-acidified endosomes in the vacuolar pathway ${ }^{30}$ have been reported to support crosspresentation. Phagocytosis and macropinocytosis typically result in the internalization of large quantities of an antigenic material that may also favor cross-presentation and priming of $\mathrm{CD}^{+}$ T cells. ${ }^{30,64}$ Although some of the HA-specific immunofluorescence was lost shortly after pulsing human MDMs with H1-VLPs, a substantial portion of the initial fluorescence was still detectable at $45 \mathrm{~min}$ and the ICCS analysis revealed striking increases in HA fluorescence intensity, cluster density, and aggregation in the endosomes. The trafficking of the HA delivered on the plantderived VLPs therefore appeared to be bidirectional: with a small portion moving rapidly into the high-degradative late endosome/ endolysosome pathway (similar to soluble HA) while a substantial amount was retained in low-degradative compartments. At $45 \mathrm{~min}$, both immunostaining and ICCS analysis demonstrated increasing colocalization of the VLP HA with both Rab5 ${ }^{+}$(early static) and Rab11 $1^{+}$(recycling) low-degradative endosomes. ${ }^{26,63}$ Nair-Gupta et al. have reported that recycling Rab11a ${ }^{+}$endosomes represent a major intracellular pool for MHC I molecules, ${ }^{29}$ and colocalization of the VLP-delivered HA with MHC I in these low-degradative compartments increased almost 3-fold between 15 and $45 \mathrm{~min}$ after the MDMs were pulsed. Thus, the $45 \mathrm{~min}$ exposure allowed us to detect endosomal degradation of the soluble HA and entry of the HA delivered by VLP into the static early/recycling endosomal compartments. Although the long-term kinetics of the disappearance of the VLP-delivered HA in the MDMs would be of some interest, it was not among the original goals of this work.

Although our imaging studies provided evidence for HA-VLP delivery into endosomal compartments that favor antigen crosspresentation, direct proof of HA processing by the human MDMs leading to the generation of MHC I-associated peptides was missing. MS-based immunopeptidome studies have historically required billions of cells to obtain sufficient numbers of $\mathrm{MHC}$ molecules for efficient detection of MHC-associated peptides. ${ }^{65}$ One consequence of this technical limitation is that most MSbased immunopeptidome work has focused on immortalized cell lines or animal cells. ${ }^{66,67}$ Recent improvements in MS technology combined with nano-flow chromatography now offer better sensitivity for the detection of MHC-associated peptides from relatively small numbers of cells. In our study, the use of a recently developed nanospray ion source with a constant flow of dopant gas permitted enhanced ionization and more efficient detection of MHC I peptides from only 3-10 million MDMs. The Maxis II mass spectrometer used in this study also has a unique hardware configuration that allows very high transmission of peptides into the collision cell enabling the detection of a wide range of peptides of various lengths. ${ }^{68}$ Our data unambiguously show that cross-presentation of HA peptides by human macrophages is possible when the HA is delivered by plant-derived VLPs. So far, MHC I-restricted presentation of influenza virus-derived peptides was thought to require infection or administration of the live attenuated vaccine, involving viral replication.,

Internal (structural) proteins of influenza viruses (i.e., nucleoprotein, polymerase basic protein 1, matrix protein 1) are thought the principle targets of the human $\mathrm{CD}^{+} \mathrm{T}$ cell response ${ }^{3}$ and only a limited number of influenza HA-derived MHC I-restricted peptides have been reported to date. ${ }^{69,70}$ We were therefore surprised to find $115 \mathrm{HA}$-derived peptides from the VLP-pulsed MDMs, 8 of which were identified in more than one donor (Supplementary Table 2). A substantial number of peptides arose from the stem region of HA molecule, raising the possibility that they may be relatively well-conserved across different viral strains with the potential to provide cross-protective immunity. Among the previously described $17 \mathrm{HA}$-derived human MHC I-restricted epitopes, ${ }^{71} 4$ peptides (23\%) were fully overlapping with up to three unique AA sequences identified in our study. Since we used unfractionated cell lysates containing MHC I molecules from the cell surface as well as those present in endosomes and the ER-Golgi compartment, it was not unexpected that many of the HA-derived peptides identified were longer than the 8-10 AA thought to be optimal for MHC I loading. ${ }^{30}$ It is likely that the longer peptides immunoprecipitated with $\mathrm{MHC}$ I were destined either for further trimming to achieve a better fit in the MHC I peptide binging groove or for degradation. ${ }^{31,72}$ The differences between donors in the number of MHC I-associated HA-derived peptides identified (i.e., fewer in donor $\mathrm{B}$ and more in donor $\mathrm{D}$ ) could be attributable to individual and (possibly) racial, gender, or age characteristics. Together, the imaging and MS results presented herein collectively suggest that influenza HA delivery to human MDMs in the form of VLPs can result in crosspresentation. It is noteworthy that VLPs produced using other platforms have been previously shown to facilitate antigen crosspresentation. $^{73-75}$ 
The potential for VLP-based vaccines to induce $C D 8^{+} T$ cells has been demonstrated in mice ${ }^{18}$ and studies are on-going to determine whether or not similar responses can be elicited in humans. Although conserved T cell epitopes from influenza core proteins have attracted the most attention to date, ${ }^{3,70}$ several human $\mathrm{CD}^{+}$epitopes have been identified in the HA proteins of both seasonal and avian influenza strains ${ }^{76,77}$ and our data suggest that many more may exist. A non-living vaccine that can induce strong antibody production as well as both $\mathrm{CD}^{+}$and $\mathrm{CD}^{+} \mathrm{T}$ cell responses might provide an added layer of protection against serious influenza virus infections compared to antibodies alone. $^{78}$

\section{METHODS}

\section{Plant-made VLP bearing influenza hemagglutinin}

The VLPs produced in N. benthamiana were kindly provided by Medicago Inc. (Quebec, QC). Plant-derived VLPs are readily available from the corresponding author upon reasonable request. The influenza HA protein was based on the sequence of A/California/07/2009 H1N1 virus. Recombinant soluble H1 protein (Immune Technology, New York, NY, Catalogue No. IT-003-SW12 $\Delta$ TMp) of the same influenza strain and influenza A (H1N1) 2009 monovalent split vaccine (Sanofi Pasteur Inc., Swiftwater, PA) were used as comparators. The HA concentration in H1-VLP and soluble HA stocks was provided by the manufacturer based on the United States Food and Drug Administration (FDA)-validated enzymelinked immunosorbent assay (ELISA) and an optical density (OD) $280 \mathrm{~nm}$ absorbance using absorbance coefficient established for the HA AA sequence, respectively.

\section{Gel electrophoresis and immunoblot analysis}

$\mathrm{H} 1-\mathrm{VLP}$ and soluble $\mathrm{H} 1$ protein samples ( 2.5 and $5 \mu \mathrm{g}$ per sample) were separated on Bis-Tris NuPAGE Gradient gel 4-12\% (Life Technologies, Carlsbad, CA) under reducing conditions followed by Coomassie blue (Life Technologies) staining. For immunoblot analysis, $0.5 \mu \mathrm{g}$ of each HA protein per sample was separated on the gel and transferred onto nitrocellulose membranes. Membranes were blocked in 5\% milk-0.05\% Tween-20 in phosphate buffer saline for $1 \mathrm{~h}$ at $22^{\circ} \mathrm{C}$ and incubated overnight at $4{ }^{\circ} \mathrm{C}$ with rabbit polyclonal anti-H1 antibody (Catalogue No. IT-003-0011, Immune Technology, New York, NY) at a 1:250 dilution, or mouse monoclonal anti-H1 antibody (clone IVC102, Catalogue No. C86304M, Meridian Life Science, Memphis, TN) at a 1:2000 dilution. Membranes were incubated for $1 \mathrm{~h}$ at $22^{\circ} \mathrm{C}$ with horseradish peroxidase-conjugated donkey anti-rabbit IgG (1:8000 dilution, Catalogue No. NA934V) or sheep antimouse IgG (1:20,000 dilution, Catalogue No. NA931V) antibodies, both from GE Healthcare Life Sciences, Uppsala, Sweden, respectively. Bands were developed using Super Signal West Pico Plus reagent (Pierce Biotechnology, Rockford, IL). Coomassie blue-stained or chemiluminescence bands were imaged using ChemiDoc ${ }^{\mathrm{TM}}$ XRS + System (Bio-Rad Laboratories, Hercules, CA) and X-ray films. All blots derived from the same experiment and were processed in parallel. Full, un-cropped images of all blots, including full molecular weight markers, are provided in Supplementary Fig. 1a-c. The total protein concentrations in the H1-VLP and soluble $\mathrm{H} 1$ stocks was measured using Pierce ${ }^{\mathrm{TM}} \mathrm{BCA}$ Protein Assay Kit (Pierce Biotechnology) according to the manufacturer's instructions.

\section{Monocyte-derived macrophages (MDMs)}

MDMs were differentiated from human peripheral blood mononuclear cells (PBMCs) isolated from healthy donors between the ages of 23 and 47 . All studies with human cells were carried out with approval from the Research Ethics Committee of the McGill University Health Centre. Written informed consent was obtained from all donors prior to blood drawing. PBMCs were separated from whole blood by centrifugation using SepMate-50 tubes (STEMCELL, Vancouver, BC). Monocytes were isolated by negative selection using magnetic microbeads according to the manufacturer's instructions (EasySep Human Monocyte Enrichment Kit, STEMCELL). Monocytes were cultured in Roswell Park Memorial Institute (RPMI)- 1640 with $50 \mathrm{IU} / \mathrm{mL}$ penicillin, $50 \mu \mathrm{g} / \mathrm{mL}$ streptomycin, and $10 \mathrm{mM}$ 4-(2-hydroxyethyl)-1-piperazineethanesulfonic acid (HEPES) (medium) supplemented with $10 \%$ fetal bovine serum (FBS, all from Wisent, Saint-JeanBaptiste, QC) and $20 \mathrm{ng} / \mathrm{mL}$ recombinant human macrophage colonystimulating factor (Gibco, Frederick, MD) for 7 days.
VLPs endocytosis assessment based on fluorescence dequenching VLPs were stained with DiD (Thermo Fisher Scientific, Eugene, OR) at $20 \mu \mathrm{g} / \mathrm{mL}$ for $30 \mathrm{~min}$ at $22^{\circ} \mathrm{C}$, and then purified from free dye using gel filtration columns (PD MiniTrap G-25, GE Healthcare, Buckinghamshire, UK). MDMs were detached from plastic plate surface using Accutase Cell Detachment Solution (BioLegend, San Diego, CA) and plated on 96-well Nunclon Delta black flat-bottom plates (Thermo Fisher Scientific, Roskilde, Denmark) at $5 \times 10^{4}$ cells/well. The following day, MDMs were exposed to DiD-labeled VLPs (HA concentration $15.0 \mu \mathrm{g} / \mathrm{mL}$ ) at $4{ }^{\circ} \mathrm{C}$ for $1 \mathrm{~h}$. Endocytosis inhibitors were applied in ice-cold medium supplemented with $10 \% \mathrm{FBS}$ : dynasore hydrate $50 \mu \mathrm{M}$, genistein $200 \mu \mathrm{M}$, amiloride hydrochloride $1 \mathrm{mM}$, cytochalasin D $4 \mu \mathrm{M}$ (all from Sigma-Aldrich, St. Louis, MO). DiD fluorescence was measured with pre-heated $\left(37^{\circ} \mathrm{C}\right)$ spectrophotometer (Infinite 200 PRO, Tecan, Männedorf, Switzerland) at 15-min intervals over $2 \mathrm{~h}$. Fusion efficiency was determined following the addition of Triton $\mathrm{X}$ 100 (Sigma-Aldrich) to each well (final concentration 1\%) to obtain full DiD dequenching.

Immunostaining and confocal microscopy

MDMs were exposed to H1-VLPs, soluble H1 or influenza A (H1N1) 2009 monovalent split vaccine at concentrations 15 or $5 \mu \mathrm{g} / \mathrm{mL}$ (by HA content) in $5 \% \mathrm{CO}_{2}$ incubator at $37^{\circ} \mathrm{C}$ for $5,10,15$, or $45 \mathrm{~min}$. Endocytosis inhibitor chlorpromazine hydrochloride $(10 \mu \mathrm{g} / \mathrm{mL}$, Sigma-Aldrich) or genistein $(200 \mu \mathrm{M})$ was applied 30 min prior to adding H1-VLPs or soluble H1, and the duration of HA exposure was $15 \mathrm{~min}$. Transferrin (human) CF568 conjugate (Biotium, Fremont, CA) was mixed with either $\mathrm{H} 1$-VLPs or soluble $\mathrm{H} 1$ (both transferrin and $\mathrm{HA}$ concentrations $15 \mu \mathrm{g} / \mathrm{mL}$ ), and the mixture was applied to the MDMs for $15 \mathrm{~min}\left(5 \% \mathrm{CO}_{2}, 37^{\circ} \mathrm{C}\right)$. In the pulse-exposure experiments, MDMs were exposed to H1-VLPs or soluble $\mathrm{H} 1$ for $15 \mathrm{~min}$, and then the supernatant was replaced by medium and kept for another $30 \mathrm{~min}$ at $5 \% \mathrm{CO}_{2}$ and $37^{\circ} \mathrm{C}$. MDMs were fixed with $4 \%$ methanol-free formaldehyde (Thermo Fisher Scientific, Rockford, IL), blocked and permeabilized with $5 \%$ goat and $5 \%$ donkey serum (both from EMD Millipore Corporation, Darmstadt, Germany) in 0.3\% Triton X-100. HA immunostaining was performed using mouse anti-H1 antibody (diluted 1:200, clone IVC102, Catalogue No. C86304M, Meridian Life Science, Memphis, TN). Endosomal proteins were visualized with rabbit anti-Rab5 (diluted 1:200, clone C8B1, Catalogue No. 3547), anti-Rab7 (diluted 1:100, clone D95F2, Catalogue No. 9367), anti-Rab11 (diluted 1:100, clone D4F5, Catalogue No. 5589) antibodies (all from Cell Signaling Technology, Danvers, MA), anti-HLA-DPB1 (diluted 1:100, clone EPR11226, Catalogue No. ab157210, Abcam, Cambridge, MA) or anti-HLA-A antibody (diluted 1:250, clone EP1395Y, Catalogue No. GTX61514, GeneTex, Irvine, CA). Secondary donkey anti-mouse Alexa Fluor 488 (diluted 1:1000, Catalogue No. A21202) and goat anti-rabbit Alexa Fluor 647 antibodies (diluted 1:1000, Catalogue No. A21245, both from Thermo Fisher Scientific, Rockford, IL) were applied for $1 \mathrm{~h}$ at $22^{\circ} \mathrm{C}$. NucBlue Live ReadyProbes Reagent-4',6-diamidino-2-phenylindole (DAPI, Thermo Fisher Scientific) used to stain cell nuclei. A laser scanning confocal microscope (Zeiss LSM780, RI-MUHC Molecular Imaging Core Facility, Montreal, QC) was used in all imaging experiments. Fluorescence intensity after cell-based segmentation of the images was quantified using ImageJ software. ${ }^{79}$

\section{Quantitative image analysis}

The fluorescence fluctuation analysis method ICCS was applied to evaluate the $\mathrm{HA}$ endosomal distribution and the degree of $\mathrm{HA}$ colocalization with endosomal proteins. In brief, ICCS measures molecular concentrations and interaction fractions based on correlation analysis of fluorescence fluctuations detected from imaged biomolecules as a function of space across an image. Automatic thresholding of fluorescence intensity via Otsu's method was applied to identify the high intensity HA-positive endosomal area in images. The negative control (no HA) samples were not suitable for ICCS since they did not display the HA fluorescence needed to segment the HA-positive endosomes for analysis. The number of HA particles per $\mu \mathrm{m}^{2}$, the HA fluorescence intensity and the aggregation state of HA particles in the HA-positive endosomal area per cell can be calculated from the spatial autocorrelation function of the fluorescence intensity fluctuations in an image from a single detection channel. Particles formed by endosomal proteins of interest (Rab5, Rab7, Rab11, MHC I, and MHC II) were also identified.

To evaluate the colocalization between HA and specific endosomal proteins, we calculated the spatial cross-correlation function from the fluorescence intensity fluctuations between images recorded in two 
different wavelength detections channels (expressed as (i) number of colocalized HA particles per $\mu \mathrm{m}^{2}$ and (ii) fraction of interacting $\mathrm{HA}$ particles) in two-color images.

\section{Transmission electron microscopy (TEM)}

MDMs were exposed to H1-VLPs or soluble $\mathrm{H} 1$ at concentration $15 \mu \mathrm{g} / \mathrm{mL}$ (by $\mathrm{HA}$ content) in $5 \% \mathrm{CO}_{2}$ incubator at $37^{\circ} \mathrm{C}$ for 5 or 15 min before fixation with $2.5 \%$ glutaraldehyde (EMS Inc., Hatfield, PA). Samples for nanogold immuno-labeling were initially fixed with $2 \%$ methanol-free formaldehyde $\left(15 \mathrm{~min}\right.$ at $22^{\circ} \mathrm{C}$ ) and permeabilized/blocked with $0.2 \%$ Triton X-100 plus $1 \%$ goat serum for $5 \mathrm{~min}$ on ice. Primary mouse anti-H1 (diluted 1:200, clone IVC102, Catalogue No. C86304M, Meridian Life Science), or rabbit anticlathrin (diluted 1:50, clone D3C6, Catalogue No. 4796) or anti-caveolin-1 (diluted 1:200, clone D46G3, Catalogue No. 3267) antibodies (both from Cell Signaling Technology) were applied overnight at $4{ }^{\circ} \mathrm{C}$. Secondary Alexa Fluor 647-FluoroNanogold goat anti-mouse $(2 \mu \mathrm{g} / \mathrm{mL}$, Catalogue No. 7501$)$ or Nanogold goat anti-rabbit (diluted 1:50, Catalogue No. 2004) antibodies, respectively (both from Nanoprobes, Yaphank, NY) were applied in 1\% nonfat dried milk, and then cells were fixed with $2.5 \%$ glutaraldehyde. Samples were washed with $0.1 \mathrm{M}$ sodium cacodylate (EMS Inc.). Cells were silverenhanced for $30 \mathrm{~s}$ using HQ Silver enhancement kit (Nanoprobes). Postfixation was done in $1 \%$ osmium tetroxide (EMS Inc.) containing potassium ferrocyanide (Fisher Scientific, Pittsburgh, PA). Cells were dehydrated with 0-100\% ethanol and progressively embedded with EPON 812 REPLACEMENT $^{\mathrm{TM}}$ kit (Mecalab Ltd., Montreal, QC). The samples were sectioned and imaged with a Tecnai T12 microscope (FEl Inc., Hillsboro, OR).

\section{Mass spectrometry analysis}

MDMs were exposed to either $\mathrm{H} 1$-VLPs or soluble $\mathrm{H} 1$ at $\mathrm{HA}$ concentration $15 \mu \mathrm{g} / \mathrm{mL}$ in $5 \% \mathrm{CO}_{2}$ incubator at $37^{\circ} \mathrm{C}$ for $16 \mathrm{~h}$. Cells were lysed $\left(1 \mathrm{~h}, 4^{\circ} \mathrm{C}\right)$ with a buffer containing 4\% NP-40 Surfact-Amps ${ }^{\mathrm{TM}}$ (Thermo Fisher Scientific, Rockford, IL), $50 \mathrm{mM}$ Tris- $\mathrm{HCl}(\mathrm{pH} 7.0), 150 \mathrm{mM} \mathrm{NaCl}$ and protease inhibitors (Pierce ${ }^{\mathrm{TM}}$ Protease Inhibitor Mini Tablets, Thermo Fisher Scientific). Lysate total protein concentration was determined using Pierce $^{\mathrm{TM}}$ BCA Protein Assay (Thermo Fisher Scientific). MHC I-peptide complexes were immunoprecipitated using Dynabeads ${ }^{\mathrm{TM}}$ protein $\mathrm{G}$ immunoprecipitation kit (Thermo Fisher Scientific) and anti-human HLA$A, B, C$ antibody (clone W6/32, Catalogue No. 311402, BioLegend, $10 \mu \mathrm{g}$ per $500 \mathrm{LL}$ lysate, $45 \mathrm{~min}, 4^{\circ} \mathrm{C}$ ). Dynabeads ${ }^{\mathrm{T}}$-antibody-antigen complexes were washed three times, transferred into clean tubes, and the peptides were eluted with $10 \%$ acetic acid $\left(70^{\circ} \mathrm{C}, 15 \mathrm{~min}\right)$. The immunoprecipitation eluate was cleaned on a C18 solid-phase extraction Macro Spin column (Harvard Apparatus, Holliston, MA) using water and methanol following manufacturer's instructions, and evaporated to dryness under vacuum ( $37^{\circ}$ C, $120 \mathrm{~min})$. Samples were reconstituted in $0.1 \%$ formic acid $(60 \mu \mathrm{L})$ and injected $(20 \mu \mathrm{L})$ onto a Maxis II (Bruker, Billerica, MA) high-resolution quadrupole-time of flight tandem mass spectrometer equipped with a Dionex UltiMate 3000 (Thermo Fisher Scientific, Waltham, MA) ultra-highperformance liquid chromatography (UHPLC) system using an Acclaim PepMap 300 RSLC C18 $2 \mu \mathrm{m} 100 \AA 150 \times 0.075 \mathrm{~mm}$ UHPLC column (Thermo Fisher Scientific) with water (A) and acetonitrile (B) both containing $0.1 \%$ formic acid at a flow rate of $0.3 \mu \mathrm{L} / \mathrm{min}\left(50^{\circ} \mathrm{C}\right)$. Elution gradient started at $5 \% \mathrm{~B}$, was held for $3 \mathrm{~min}$, then increased to $35 \%$ at $73 \mathrm{~min}, 55 \%$ at $90 \mathrm{~min}$, and $80 \%$ at $95 \mathrm{~min}$. MS spectra were acquired at $\mathrm{m} /$ $z$ 400-2200 and MS/MS spectra were recorded at m/z 150-2200 using collision-induced dissociation (CID) activation in Auto MS/MS mode with a collision energy of $21-55 \mathrm{eV}$ depending on precursor ion $\mathrm{m} / \mathrm{z}$ value and charge state $(z)$. lons with $z=2-5$ were preferred whereas singly charged ions were excluded. Redundant ions were also excluded for $2 \mathrm{~min}$. Acquisition time was $0.5 \mathrm{~s}$ for MS and $0.06-0.25 \mathrm{~s}$ for each MS/MS scan depending on precursor ion signal intensity, with a total cycle time of $3.0 \mathrm{~s}$. CaptiveSpray (Bruker) nanospray ionization source operated in positive mode with a capillary voltage of $1.8 \mathrm{kV}$. To enhance ionization, a continuous flow of nitrogen and vaporized acetonitrile (as dopant) was injected into the ion source during the analysis using a nanoBooster module (Bruker). Nitrogen (99.5\% pure) was used as dry gas $\left(150^{\circ} \mathrm{C}\right)$ at a flow rate of $3.0 \mathrm{~L} / \mathrm{min}$. Samples were analyzed in duplicate.

\section{Analysis of proteomics data}

Mass spectra were imported into the MaxQuant software ${ }^{80}$ and searched against an in-house influenza A virus (A/California/07/2009(H1N1)) hemagglutinin FASTA file including six UniProt-TrEMBL identifiers: C3W627, C3W5X2, 16T4Z8, R9RVT8, U3M8B4, U3M8F8. An unspecific search was conducted for peptides with a length of 5-20 residues at 0.01 falsediscovery rate (FDR). Methionine oxidation and $\mathrm{N}$-terminal acetylation were defined as variable modifications. A PEP threshold of 0.01 was set for all MaxQuant searches. To account for LC retention shifts, the "match between runs" option was enabled with a match time window of $0.7 \mathrm{~min}$ and an alignment time window of 20 min. Only hits with a protein score $>30$ were accepted.

\section{Statistical analysis}

Statistical analysis was performed using GraphPad Prism 6.0 software. Oneway analysis of variance (ANOVA) followed by Tukey's multiple comparisons post-test or Mann-Whitney test was used to examine the differences between samples. $p$-values $<0.05$ were considered statistically significant.

\section{DATA AVAILABILITY}

The datasets generated and/or analyzed during the current study are available from the corresponding author. The MS proteomics data have been deposited to the ProteomeXchange Consortium via the PRoteomics IDEntifications (PRIDE) partner repository ${ }^{81}$ with the dataset identifier PXD010519.

\section{CODE AVAILABILITY}

Custom MATLAB code used for ICCS is available from the corresponding author upon reasonable request.

\section{ACKNOWLEDGEMENTS}

This work was supported by the Canadian Institutes of Health Research (Grant \# UI301202) and the Ministère de l'Économie et de I'Innovation (MEI) of Quebec with project oversight by Génome Québec. P.W.W. acknowledges support from a Natural Sciences and Engineering Research Council of Canada (NSERC) discovery grant (Grant \# RGPIN-2017-05005). The authors thank Dr. John Presley at McGill University for helpful discussions during the planning of experiments and manuscript preparation. The authors thank the Molecular Imaging Platform of Research Institute of the McGill University Health Centre and its staff for the kind services provided.

\section{AUTHOR CONTRIBUTIONS}

A.I.M. and B.J.W. developed the conception and designed the study. The experiments and data analysis were performed by A.I.M., M.G., E.R.-L., A.A.G., I.N.C.-C. and S.C. The manuscript was drafted by A.I.M., M.G. and A.A.G. and S.P., N.C., N.L., I.R., P.W.W., M.N. and B.J.W. revised it critically and provided input for important intellectual content. All authors approved the final version of the article prior to its submission.

\section{ADDITIONAL INFORMATION}

Supplementary information accompanies the paper on the npj Vaccines website (https://doi.org/10.1038/s41541-019-0111-y).

Competing interests: B.J.W. has been principal or site investigator for vaccine trials for several manufacturers including Medicago Inc. Since 2010, B.J.W. has served as Medical Officer for Medicago Inc. B.J.W. and I.R. have held and continue to hold peerreviewed support for collaborative, basic science work with Medicago Inc. B.J.W. has received honoraria from several vaccine manufacturers for participation on Scientific Advisory Boards. A.I.M., S.P., N.C., and N.L. are current employees of Medicago Inc. The other authors declare no competing interests.

Publisher's note: Springer Nature remains neutral with regard to jurisdictional claims in published maps and institutional affiliations.

\section{REFERENCES}

1. Sridhar, S. Heterosubtypic T-cell immunity to influenza in humans: challenges for universal T-cell influenza vaccines. Front. Immunol. 7, 195 (2016).

2. Wilkinson, T. M. et al. Preexisting influenza-specific CD4 + T cells correlate with disease protection against influenza challenge in humans. Nat. Med. 18, 274-280 (2012).

3. Grant, E. J., Quinõnes-Parra, S. M., Clemens, E. B. \& Kedzierska, K. Human influenza viruses and CD8+ T cell responses. Curr. Opin. Virol. 16, 132-142 (2016).

4. Mohn, K. G.-I., Smith, I., Sjursen, H. \& Cox, R. Immune responses after live attenuated influenza vaccination. Hum. Vaccin. Immunother. 14, 571-578 (2018). 
5. Nguyen, T. H. O. et al. Perturbed $C D 8^{+} \mathrm{T}$ cell immunity across universal influenza epitopes in the elderly. J. Leukoc. Biol. 103, 321-339 (2017).

6. Bonduelle, $\mathrm{O}$. et al. Longitudinal and integrative biomodeling of effector and memory immune compartments after inactivated influenza vaccination. J. Immunol. 191, 623-631 (2013).

7. Hoft, D. F. et al. Comparisons of the humoral and cellular immune responses induced by live attenuated influenza vaccine and inactivated influenza vaccine in adults. Clin. Vaccin. Immunol. 24, e00414-e00416 (2017).

8. D'Aoust, M. A. et al. Influenza virus-like particles produced by transient expression in Nicotiana benthamiana induce a protective immune response against a lethal viral challenge in mice. Plant Biotechnol. J. 6, 930-940 (2008).

9. D'Aoust, M. A. et al. The production of hemagglutinin-based virus-like particles in plants: a rapid, efficient and safe response to pandemic influenza. Plant Biotechnol. J. 8, 607-619 (2010).

10. Lindsay, B. J. et al. Morphological characterization of a plant-made virus-like particle vaccine bearing influenza virus hemagglutinins by electron microscopy. Vaccine 36, 2147-2154 (2018).

11. Le Mauff, F. et al. Biochemical composition of haemagglutinin-based influenza virus-like particle vaccine produced by transient expression in tobacco plants. Plant Biotechnol. J. 13, 717-725 (2015).

12. Hendin, H. E. et al. Plant-made virus-like particle vaccines bearing the hemagglutinin of either seasonal $(\mathrm{H} 1)$ or avian $(\mathrm{H} 5)$ influenza have distinct patterns of interaction with human immune cells in vitro. Vaccine 35, 2592-2599 (2017).

13. Makarkov, A. I. et al. Plant-made virus-like particles bearing influenza hemagglutinin (HA) recapitulate early interactions of native influenza virions with human monocytes/macrophages. Vaccine 35, 4629-4636 (2017).

14. Hodgins, B. et al. A single intramuscular dose of a plant-made virus-like particle vaccine elicits a balanced humoral and cellular response and protects young and aged mice from influenza H1N1 virus challenge despite a modest/absent humoral response. Clin. Vaccin. Immunol. 24, 1-14 (2017).

15. Pillet, $\mathrm{S}$. et al. A plant-derived quadrivalent virus like particle influenza vaccine induces cross-reactive antibody and $\mathrm{T}$ cell response in healthy adults. Clin. Immunol. 168, 72-87 (2016).

16. Pillet, S. et al. Humoral and cell-mediated immune responses to $\mathrm{H} 5 \mathrm{~N} 1$ plant-made virus-like particle vaccine are differentially impacted by alum and GLA-SE adjuvants in a Phase 2 clinical trial. npj Vaccines 3, 3 (2018).

17. Landry, N. et al. Influenza virus-like particle vaccines made in Nicotiana benthamiana elicit durable, poly-functional and cross-reactive $T$ cell responses to influenza HA antigens. Clin. Immunol. 154, 164-177 (2014).

18. Won, S. Y. et al. Characterization of the innate stimulatory capacity of plantderived virus-like particles bearing influenza hemagglutinin. Vaccine 36, 8028-8038 (2018).

19. $\mathrm{Wu}, \mathrm{Y}$. et al. A potent broad-spectrum protective human monoclonal antibody crosslinking two haemagglutinin monomers of influenza A virus. Nat. Commun. 6 , 7708 (2015).

20. Klumperman, J. \& Raposo, G. The complex ultrastructure of the endolysosomal system. Cold Spring Harb. Perspect. Biol. 6, a016857 (2014).

21. Burgdorf, S. \& Kurts, C. Endocytosis mechanisms and the cell biology of antigen presentation. Curr. Opin. Immunol. 20, 89-95 (2008).

22. Kolin, D. L. \& Wiseman, P. W. Advances in image correlation spectroscopy: measuring number densities, aggregation states, and dynamics of fluorescently labeled macromolecules in cells. Cell Biochem. Biophys. 49, 141-164 (2007).

23. Gopal, A. A. et al. Spatially selective dissection of signal transduction in neurons grown on Netrin-1 printed nanoarrays via segmented fluorescence fluctuation analysis. ACS Nano 11, 8131-8143 (2017).

24. Gautreau, A., Oguievetskaia, K. \& Ungermann, C. Function and regulation of the endosomal fusion and fission machineries. Cold Spring Harb. Perspect. Biol. 6 , a016832 (2014).

25. Jovic, M., Sharma, M., Rahajeng, J. \& Caplan, S. The early endosome: a busy sorting station for proteins at the crossroads. Histol. Histopathol. 25, 99-112 (2010).

26. Lakadamyali, M., Rust, M. J. \& Zhuang, X. Ligands for clathrin-mediated endocytosis are differentially sorted into distinct populations of early endosomes. Cell 124, 997-1009 (2006)

27. ten Broeke, T., Wubbolts, R. \& Stoorvogel, W. MHC class II antigen presentation by dendritic cells regulated through endosomal sorting. Cold Spring Harb. Perspect. Biol. 5, a016873 (2013).

28. Donaldson, J. G., Johnson, D. L. \& Dutta, D. Rab and Arf G proteins in endosomal trafficking and cell surface homeostasis. Small GTPases 7, 247-251 (2016).

29. Nair-Gupta, P. et al. TLR signals induce phagosomal MHC-I delivery from the endosomal recycling compartment to allow cross-presentation. Cell 158, 506-521 (2014).

30. Blander, J. M. Regulation of the cell biology of antigen cross presentation. Annu. Rev. Immunol. 36, 717-753 (2018)
31. Saveanu, L. et al. Concerted peptide trimming by human ERAP1 and ERAP2 aminopeptidase complexes in the endoplasmic reticulum. Nat. Immunol. 6 689-697 (2005).

32. Duan, M., Hibbs, M. L. \& Chen, W. The contributions of lung macrophage and monocyte heterogeneity to influenza pathogenesis. Immunol. Cell Biol. 95, 225-235 (2017).

33. Sander, J. et al. Cellular differentiation of human monocytes is regulated by timedependent interleukin-4 signaling and the transcriptional regulator NCOR2. Immunity 47, 1051-1066 (2017).

34. He, W. et al. Alveolar macrophages are critical for broadly-reactive antibodymediated protection against influenza A virus in mice. Nat. Commun. 8, 846 (2017).

35. Chroboczek, J., Szurgot, I. \& Szolajska, E. Virus-like particles as vaccine. Acta Biochim. Pol. 61, 531-539 (2014)

36. Liu, J. et al. Virus like particle-based vaccines against emerging infectious disease viruses. Virol. Sin. 31, 279-287 (2016).

37. Rynda-Apple, A., Patterson, D. P. \& Douglas, T. Virus-like particles as antigenic nanomaterials for inducing protective immune responses in the lung. Nanomedicine 9, 1857-1868 (2014).

38. Yan, D., Wei, Y. Q., Guo, H. C. \& Sun, S. Q. The application of virus-like particles as vaccines and biological vehicles. Appl. Microbiol. Biotechnol. 99, 10415-10432 (2015).

39. Kang, S.-M., Kim, M.-C. \& Compans, R. W. Virus-like particles as universal influenza vaccines. Expert Rev. Vaccin. 11, 995-1007 (2012).

40. Moffat, J. M., Cheong, W. S., Villadangos, J. A., Mintern, J. D. \& Netter, H. J. Hepatitis B virus-like particles access major histocompatibility class I and II antigen presentation pathways in primary dendritic cells. Vaccine 31, 2310-2316 (2013).

41. Ponterio, E. et al. Pattern of activation of human antigen presenting cells by genotype Gll.4 norovirus virus-like particles. J. Transl. Med. 11, 127 (2013).

42. Chen, S. et al. Protection against multiple subtypes of influenza viruses by viruslike particle vaccines based on a hemagglutinin conserved epitope. Biomed. Res. Int. 2015, 901817 (2015).

43. Schmeisser, F. et al. Production and characterization of mammalian virus-like particles from modified vaccinia virus Ankara vectors expressing influenza H5N1 hemagglutinin and neuraminidase. Vaccine 30, 3413-3422 (2012).

44. Gao, X. et al. Enhanced Influenza VLP vaccines comprising matrix-2 ectodomain and nucleoprotein epitopes protects mice from lethal challenge. Antivir. Res. 98 4-11 (2013).

45. Ren, Z. et al. Intramuscular and intranasal immunization with an H7N9 influenza virus-like particle vaccine protects mice against lethal influenza virus challenge. Int. Immunopharmacol. 58, 109-116 (2018).

46. Xue, $C$. et al. Incorporation of conserved nucleoprotein into influenza virus-like particles could provoke a broad protective immune response in BALB/c mice and chickens. Virus Res. 195, 35-42 (2015).

47. Chung, K. Y. et al. ISCOMATRIX ${ }^{\mathrm{TM}}$ adjuvant promotes epitope spreading and antibody affinity maturation of influenza A H7N9 virus like particle vaccine that correlate with virus neutralization in humans. Vaccine 33, 3953-3962 (2015).

48. Cummings, J. F. et al. Safety and immunogenicity of a plant-produced recombinant monomer hemagglutinin-based influenza vaccine derived from influenza A (H1N1)pdm09 virus: a Phase 1 dose-escalation study in healthy adults. Vaccine 32, 2251-2259 (2014)

49. Low, J. G. H. et al. Safety and immunogenicity of a virus-like particle pandemic influenza A (H1N1) 2009 vaccine: results from a double-blinded, randomized Phase I clinical trial in healthy Asian volunteers. Vaccine 32, 5041-5048 (2014).

50. Huang, X., Wang, X., Zhang, J., Xia, N. \& Zhao, Q. Escherichia coli-derived virus-like particles in vaccine development. npj Vaccines 2, 1-8 (2017).

51. Kazaks, A. et al. Production and purification of chimeric $\mathrm{HBc}$ virus-like particles carrying influenza virus LAH domain as vaccine candidates. BMC Biotechnol. 17, 79 (2017).

52. Sequeira, D. P. et al. Combining stable insect cell lines with baculovirus-mediated expression for multi-HA influenza VLP production. Vaccine 36, 3112-3123 (2018).

53. Wu, C.-Y. et al. Mammalian expression of virus-like particles for advanced mimicry of authentic influenza virus. PLoS ONE 5, e9784 (2010).

54. Rodríguez-Limas, W. A., Sekar, K. \& Tyo, K. E. J. Virus-like particles: the future of microbial factories and cell-free systems as platforms for vaccine development. Curr. Opin. Biotechnol. 24, 1089-1093 (2013).

55. Scotti, N. \& Rybicki, E. P. Virus-like particles produced in plants as potential vaccines. Expert Rev. Vaccin. 12, 211-224 (2013).

56. Chen, Q. \& Lai, H. Plant-derived virus-like particles as vaccines. Hum. Vaccin. Immunother. 9, 26-49 (2013).

57. Marsian, J. \& Lomonossoff, G. P. Molecular pharming-VLPs made in plants. Curr. Opin. Biotechnol. 37, 201-206 (2016)

58. Steele, J. F. C. et al. Synthetic plant virology for nanobiotechnology and nanomedicine. Wiley Interdiscip. Rev. Nanomed. Nanobiotechnol. 9, 1-18 (2017) 
59. Ravin, N. V. et al. Plant-produced recombinant influenza vaccine based on viruslike $\mathrm{HBc}$ particles carrying an extracellular domain of M2 protein. Biochemistry 77, 33-40 (2012).

60. Shoji, Y. et al. Immunogenicity of H1N1 influenza virus-like particles produced in Nicotiana benthamiana. Hum. Vaccin. Immunother. 11, 118-123 (2015).

61. Scott, C. C., Vacca, F. \& Gruenberg, J. Endosome maturation, transport and functions. Semin. Cell Dev. Biol. 31, 2-10 (2014).

62. Kiss, A. L. Caveolae and the regulation of endocytosis. Adv. Exp. Med. Biol. 729, 14-28 (2012).

63. Tanaka, T. et al. Heat shock protein 90 targets a chaperoned peptide to the static early endosome for efficient cross-presentation by human dendritic cells. Cancer Sci. 106, 18-24 (2015).

64. Hari, A. et al. Redirecting soluble antigen for MHC class I cross-presentation during phagocytosis. Eur. J. Immunol. 45, 383-395 (2015).

65. Caron, E. et al. Analysis of major histocompatibility complex (MHC) immunopeptidomes using mass spectrometry. Mol. Cell. Proteomics 14, 3105-3117 (2015).

66. Karunakaran, K. P. et al. Identification of MHC-bound peptides from dendritic cells infected with Salmonella enterica strain SL1344: implications for a nontyphoidal Salmonella vaccine. J. Proteome Res. 16, 298-306 (2017).

67. Mobbs, J. I. et al. The molecular basis for peptide repertoire selection in the human leucocyte antigen (HLA) $\mathrm{C} * 06: 02$ molecule. J. Biol. Chem. 292, 17203-17215 (2017).

68. Beck, S. et al. The Impact II, a very high-resolution quadrupole time-of-flight instrument (QTOF) for deep shotgun proteomics. Mol. Cell. Proteomics 14, 2014-2029 (2015).

69. Keskin, D. B. et al. Physical detection of influenza A epitopes identifies a stealth subset on human lung epithelium evading natural CD8 immunity. Proc. Natl. Acad. Sci. 112, 2151-2156 (2015).

70. Wahl, A. et al. HLA class I molecules consistently present internal influenza epitopes. Proc. Natl. Acad. Sci. 106, 540-545 (2009).

71. National Institute of Allergy and Infectious Diseases. Free epitope database and prediction resource (2018). Available at http://www.iedb.org. Accessed 4 June 2018.

72. Saveanu, L. et al. IRAP identifies an endosomal compartment required for MHC class i cross-presentation. Science 325, 213-217 (2009).

73. Guo, L. et al. Enhancement of mucosal immune responses by chimeric influenza HA/SHIV virus-like particles. Virology 313, 502-513 (2003).
74. Hemann, E. A., Kang, S.-M. \& Legge, K. L. Protective CD8 T cell-mediated immunity against influenza $A$ virus infection following influenza virus-like particle vaccination. J. Immunol. 191, 2486-2494 (2013).

75. Song, $\mathrm{H}$. et al. In vitro stimulation of human influenza-specific CD8+ T cells by dendritic cells pulsed with an influenza virus-like particle (VLP) vaccine. Vaccine 28, 5524-5532 (2010).

76. Qiu, X. et al. Lineage-specific epitope profiles for HPAI H5 pre-pandemic vaccine selection and evaluation. Influenza Other Respir. Viruses 11, 445-456 (2017).

77. Muralidharan, A. et al. Identification of immunodominant CD8 epitope in the stalk domain of influenza B viral hemagglutinin. Biochem. Biophys. Res. Commun. 502, 226-231 (2018).

78. Schmidt, M. E. \& Varga, S. M. The CD8 T cell response to respiratory virus infections. Front. Immunol. 9, 678 (2018).

79. Schneider, C. A., Rasband, W. S. \& Eliceiri, K. W. NIH Image to ImageJ: 25 years of image analysis. Nat. Methods 9, 671-675 (2012).

80. Cox, J. \& Mann, M. MaxQuant enables high peptide identification rates, individualized p.p.b.-range mass accuracies and proteome-wide protein quantification. Nat. Biotechnol. 26, 1367-1372 (2008).

81. Vizcaíno, J. A. et al. 2016 update of the PRIDE database and its related tools. Nucleic Acids Res. 44, D447-D456 (2016).

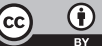

Open Access This article is licensed under a Creative Commons Attribution 4.0 International License, which permits use, sharing, adaptation, distribution and reproduction in any medium or format, as long as you give appropriate credit to the original author(s) and the source, provide a link to the Creative Commons license, and indicate if changes were made. The images or other third party material in this article are included in the article's Creative Commons license, unless indicated otherwise in a credit line to the material. If material is not included in the article's Creative Commons license and your intended use is not permitted by statutory regulation or exceeds the permitted use, you will need to obtain permission directly from the copyright holder. To view a copy of this license, visit http://creativecommons. org/licenses/by/4.0/.

(c) The Author(s) 2019 